\title{
Dermal Fibroblast Cells: Biology and Function in Skin Regeneration
}

\author{
Mohammad Ali Nilforoushzadeh, ${ }^{1}$ Hamid Reza Ahmadi Ashtiani, ${ }^{2}$ Fariba Jaffary, ${ }^{3}$ Faeze Jahangiri, ${ }^{1}$ \\ Nahid Nikkhah, ${ }^{1}$ Mona Mahmoudbeyk, ${ }^{1}$ Maryam Fard, ${ }^{1}$ Zibajaber Ansari, ${ }^{1}$ and Sona Zare ${ }^{1,4,}{ }^{*}$ \\ ${ }^{1}$ Skin and Stem Cell Research Center, Tehran University of Medical Sciences, Tehran, Iran \\ ${ }^{2}$ Department of Basic Sciences, Faculty of Pharmacy, Pharmaceutical Sciences Branch, Islamic Azad University (IAUPS), Tehran, Iran \\ ${ }^{3}$ Skin Diseases and Leishmaniasis Research Center, Isfahan University of Medical Sciences, Isfahan, Iran \\ ${ }^{4}$ Department of Biology, Faculty of Basic Science, Islamic Azad University, Hamedan Branch, Hamedan, Iran \\ "Corresponding author: Sona Zare, Skin and Stem Cell Research Center, Tehran University of Medical Sciences, Tehran, Iran. Tel: +98-2122215558, Fax: +98-2122239264, E-mail: \\ s-zare@farabi.tums.ac.ir
}

Received 2017 April 10; Revised 2017 May 09; Accepted 2017 June 12.

\begin{abstract}
Fibroblasts are mesenchymal cells, which play an important role in epithelial-mesenchymal reactions. These cells also play a key role in epidermal proliferation and differentiation and cellular matrix formation through secretion of different growth factors and cytokines. Cell therapy is a new treatment for skin diseases. In fact, conditions, such as burns, diabetic wounds, scars, and aging skin, have been treated with these cells. In cell therapy, samples obtained from the patient's skin are isolated and cultured; then, the cells are injected into damaged tissues. In recent years, several clinical trials have been conducted in this area, and numerous studies have been published. In this review, we aimed to investigate the biology and function of these cells in the treatment of skin diseases.
\end{abstract}

Keywords: Fibroblast Cells, Cell Therapy, Scar, Wound, Rejuvenation

\section{Context}

In recent years, owing to extensive research and advances in the field of cell therapy, a new approach has been developed in the treatment of multiple diseases, using autologous and allogeneic cells. Today, research centers and knowledge-based organizations are making great efforts to use these cells in the treatment of various diseases around the world. Knowledge of cell therapy and its application have many potential advantages. Stem cells and somatic cells can be used in transplantation of different types of tissues and can stimulate other cells to repair through various cellular mechanisms, such as intracellular interactions.

Use of functional cells with therapeutic efficacy can restore tissue function through direct contribution of cells to the target tissue or carrier complex and affect cellular symptoms without direct involvement. These outstanding features highlight the importance of cell therapy. Researchers in medical sciences believe that use of cell therapy, along with gene therapy, can influence the occurrence of diseases in the future $(1,2)$.

New treatments for skin diseases are among tremendous advances in the field of cell therapy. Introduction of cellular technology, especially application of autolo- gous stem cells in the treatment of skin diseases, has drawn considerable attention and anticipation in this field over the past decades. Accordingly, leading research centers around the world have initiated various projects in this area, resulting in the dramatic development of stem cell technology and strategic knowledge in industrialized countries. With this background in mind, the present study aimed to discuss the biological and functional properties of fibroblastic cell products (3).

\section{Evidence Acquisition}

\subsection{Skin Structure}

The barrier system comprises the skin, as the largest body organ, and its appendages, accounting for $16 \%$ of body weight. The skin contains epidermis and dermis; dermis is recognized as the inner layer. In addition to covering the underlying soft tissues, the skin also has other properties, such as protection against injuries, bacterial attacks, and water loss. Moreover, adjustment of body temperature and receiving various stimuli from the environment (such as touch, temperature, and pain) are among other characteristics of the skin. Other roles of the skin include secretion induction of sweat glands and UV absorption from sunlight to produce vitamin D (4). 


\subsection{Structure of the Dermis}

The dermis, lying directly under the epidermis, is derived from the mesoderm lineage and is divided into two layers: 1, a wavy layer which is a loose and superficial papillary layer; and 2, a reticular layer which is denser and deeper. The dermis includes irregular, dense, and collagenous connective tissues. It mainly contains collagen type I strands and elastic fibers, which support the epidermis and bind the skin to the underside of hypothalamus (fascia). The dermal thickness varies from $0.6 \mathrm{~mm}$ in the eyelid to approximately $3 \mathrm{~mm}$ in the palm of hands and soles of feet. However, there is no exact boundary in the contact area of the dermis with the fascia. Normally, the dermis is thicker in men compared to women; in addition, it is thicker in the dorsal regions of the body (Figure 1) (5-7).

\subsection{Embryonic Development of the Dermis}

The dermis is derived from the underlying mesenchymal surface ectoderm. The mesenchymal tissue is differentiated from the dorsal part of dermal connective tissues and is derived from the somatostatic dermatome. This tissue is formed in the abdominal region of the trunk, whereas organs develop from the somatic layer of lateral mesoderm. In the face and neck, nerve rupture contributes to the formation of dermal layer. The Wnt signal, through $\beta$-catenin signal, causes Dermo-1, a dermal marker in most adjacent cells, to form ectoderm in the myometrium.

Dermis-derived mesenchymal cell debris is initially connected by tight joints. These dermal precursor cells initially secrete an intercellular liquid-rich matrix of glycogen and hyaluronic acid. In the eleventh week, the dermis undergoes a distinct condition in which mesenchymal cells differentiate into fibroblasts. Also, an intercellular matrix of fibrosis is formed, in which the main strands include collagen I, collagen III, and elastic fibers.

The interaction between the dermis and epidermis is not only necessary for multiplication of epidermis, but is also essential for formation and regional variations of the epidermis. For instance, in the very early stages of development, the epidermis in the palm of hands and foot sole is thicker than other areas, and hair is not formed in these regions. Studies have demonstrated that type of epidermis is affected by the dermal layer.

Once epidermal rings are formed, the dermis penetrates into the epidermis and creates dermal rhizomes, which collapse upon each other, similar to a zipper. In some of these rings, capillary loops (i.e., endothelial tubes) feed the epidermis. In the eighteenth week of development, nerves enter the epidermis and dermis, reflex arches are completed, and the fetus can respond to pressure and shock. Initially, dermal blood vessels are formed as endothelium-lined structures, derived from the mesenchyme. Additionally, with the development of skin, new capillaries are formed from arteries in the dermis (angiogenesis) at the end of the fifth week.

When myoblasts differentiate into the surrounding mesenchyme, some of the new capillaries produce a muscle cover, resulting in the formation of shear cells and arteries. In contrast, capillaries, which return blood, absorb the muscle cover and move into veins. In general, when new blood vessels are formed, some vessels are disrupted. At the end of the first three months of pregnancy, dermal vessels fully develop (8-10).

\subsection{Dermal Classification}

The dermis is composed of a top papillary layer and a denser and thicker tricolored layer. The surface papillary layer of the dermis is rugged and composed of connective tissues with type III collagens (reticular strands); the elastic strands are also arranged in a loose network. Anthrax fibrils, which consist of type VII collagens, extend from the base blade to the papillary layer and attach the epidermis to the dermis.

The papillary layer contains fibroblasts, macrophages, plasma cells, mast cells, and other commonly utilized connective tissue cells. The papillary layer contains a large number of capillary arches, extending to the epidermaldermal contact point. These capillaries regulate body temperature and feed epidermal cells without vessels. Meissner's bodies are both pear- and capsule-shaped and are found in some dermal papillae. Meissner's bodies are also spin-offs, which respond to the slight destruction of the epidermis. These receptors are located in certain skin areas, such as lips, external genital organs, and nipples, and are indeed more sensitive to stimuli. Another capsule mechanoprocessor is known as "bubble", which is formed in the papillary layer. Although this receptor was initially thought to respond to cold temperature, its function remains unclear.

The contact area between the papillary layer and reticular layer of the dermis cannot be distinguished, as these layers are located next to each other. In general, the reticular layer consists of irregular, dense, and collagenous connective tissues, possessing thick type I collagens with iodine. These strings are arranged together in large batches, which are mostly parallel to the surface of the skin. They include networks of thick elastic strings, formed alongside collagen strings; these elastic strands are at maximum level near sebaceous and sweat glands.

Proteoglycans, which are rich in dermatan sulfate, fill the interstitial regions of the reticular layer. Cells in the reticular layer are more dispersed than those in the papillary layer and include fibroblasts, mast cells, lymphocytes, 


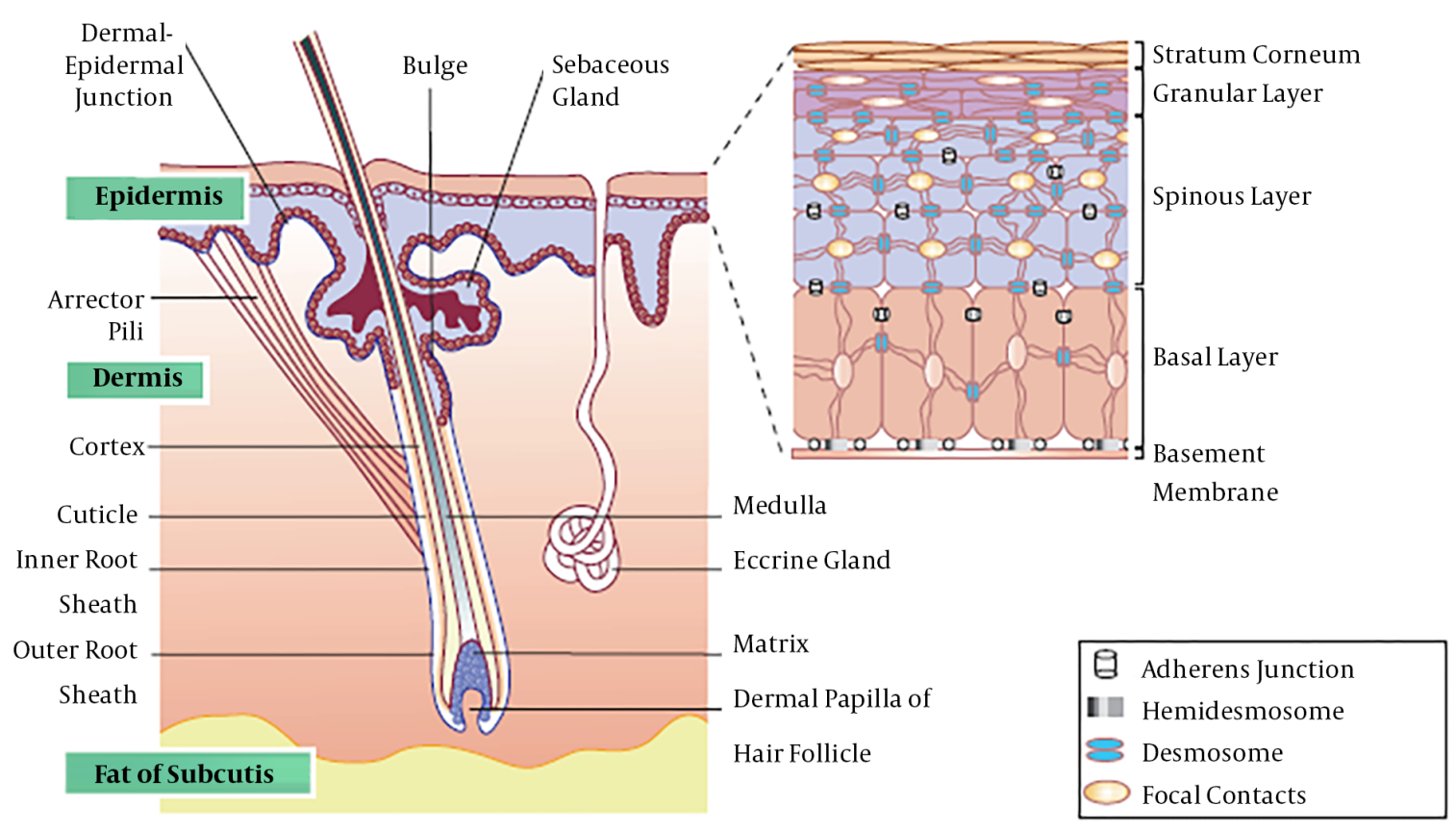

Figure 1. The skin and its appendages (7)

and macrophages. Occasionally, fat cells are observed in the deep parts of the reticular layer. Sweat glands, sebaceous glands, and hair follicles are derived from the epidermis, penetrate into the dermis and hypoderm during embryogenesis, and remain there permanently. Smooth muscle cell groups are found in the deeper regions of the reticular layer in specific areas, such as the penis and scrotum skin and nasal arthritis (11-13).

\subsection{Biology of Fibroblasts}

Fibroblasts are the most abundant cells in connective tissues. They primarily contribute to the secretion of extrarenal-matrix prophylaxis material to maintain the structural integrity of connective tissues. They secrete the necessary precursors for the production of all extracellular matrix compounds, including the base material and its strands. Similar to other connective tissue cells, fibroblasts are derived from the mesenchyme. The intermediate filament secretes a protein called vimentin, which is used as an indicator for identification of mesodermal origins.

Epithelial cells due to epithelial-mesenchymal transition can convert into fibroblasts and vice versa. Furthermore, fibroblasts can be converted to epithelial cells under certain conditions. Mesenchymal-epithelial transition is also known as mesenchymal-epithelial transition. Fibroblasts consist of collagen, glycosaminoglycan, lattice and elastic fibers, glycoproteins in the exocrine matrix, and thymic stromal lymphopoietin cytokines; growing fibroblasts also divide and form the base material.

Unlike epithelial cells, single-stranded fibroblasts include cells, which do not form broad tissues and are not limited to the basal layer by unipolar connection. Moreover, they sometimes contribute to the formation of basal layer. For instance, intestinal myofibroblasts secrete $\alpha-2$ chain-carrying laminin components; yet, follicular areas are only absent in the epithelium. In contrast with epithelial cells, fibroblasts can individually migrate to the substrate layer. At epithelial surfaces of the inner surface of body organs, fibroblasts and other related connective tissues determine the exterior appearance and shape of these structures (Figure 2) (14-17).

\section{Results}

\subsection{Function of Fibroblasts}

In recent years, various studies have been conducted to verify the capacity of fibroblasts to regenerate the skin structure. Tissue damage induces fibroblasts and mitosis. On the other hand, in injured areas, these cells initiate cellular signaling processes, proliferate and migrate to the wound, contribute to extracellular matrix production, and 


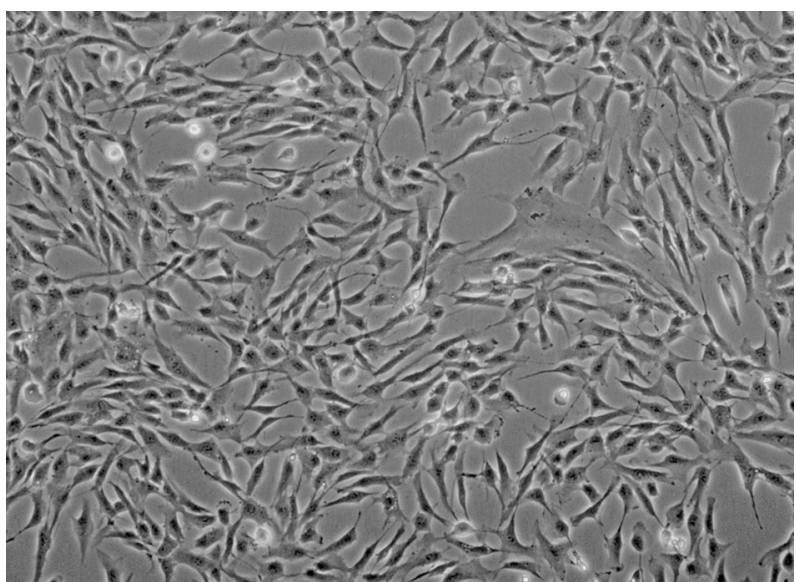

Figure 2. Fibroblast cells from the human skin $(\times 100)$

repair tissues. Extracellular matrix proteins act as a scaffold for migration of inflammatory cells and production of granular tissues.

Granular tissue formation provides a temporary substrate for strengthening epithelial tissues by keratinocytes. With the formation of fibroblasts, they produce contractile elements for ulcer closure and produce type I collagens in subsequent phases to accelerate the healing process. Collagen is the most common form of extracellular envelope, which forms fibers in an extracellular environment; therefore, it determines the shape of tissues. Collagen is stored as a precursor in fibroblastic cells and is secreted to the extracellular medium. The injected fibroblasts stimulate the production of extracellular matrix proteins or skin tissue regeneration.

In 2011, Han Kim et al. stated that mesenchymal stem cells, along with collagen gel, improve wound healing. In their study, three areas in the dorsal area of rats were punched; one part received collagen gel alone, one part received collagen gel in addition to mesenchymal stem cells, and one part received none as the control. They observed that mesenchymal stem cells could significantly increase wound healing by activating matrix metalloproteinase 9 and vascular endothelial growth factor. The literature suggests the use of these cells in various therapeutic interventions, such as treatment of ulcers due to surgery or burns, chronic ulcers, treatment resistance in diabetic ulcers, and shallow or deep wrinkles of the skin $(18,19)$.

\subsection{Comparison of Fibroblastic Markers with Other Mesenchy- mal Cells}

Fibroblasts and mesenchymal stem cells express different genes. These variations are attributed to specific receptors and different markers in comparison with other

\begin{tabular}{|c|c|c|}
\hline Cell Surface Markers & Mesenchymal Stem Cells & Fibroblasts \\
\hline CD9 & + & + \\
\hline CD10 & \pm & + \\
\hline CD13 & + & + \\
\hline CD26 & Variable & + \\
\hline CD29 & + & + \\
\hline CD44 & + & + \\
\hline CD49a & + & + \\
\hline CD49b & + & + \\
\hline CD54 & + & + \\
\hline CD71 & + & + \\
\hline CD73 & + & + \\
\hline CD9o & + & + \\
\hline CD105 & + & + \\
\hline CD106 & + & - \\
\hline CD138 & Variable & Variable \\
\hline CD146 & + & \pm \\
\hline CD164 & + & + \\
\hline CD166 & + & + \\
\hline ITGA 11 & + & - \\
\hline CD11a & - & - \\
\hline CD14 & - & - \\
\hline CD16 & - & - \\
\hline CD31 & - & - \\
\hline CD33 & - & - \\
\hline CD43 & - & - \\
\hline CD45 & - & - \\
\hline CD86 & - & - \\
\hline CD117 & - & - \\
\hline CD133 & - & - \\
\hline CD184 & - & - \\
\hline
\end{tabular}

cells. As a result, fibroblasts and mesenchymal stem cells are used for therapeutic and research purposes. For instance, markers of CD26 and CD10 expression vary or are not expressed in the majority of mesenchymal cells. However, in fibroblasts, the markers show adequate expression and are used to identify these cells (Table 1) $(20,21)$.

\section{Conclusion}

In addition to phenotypic and genetic similarities between fibroblasts and mesenchymal stem cells, they both 
exert immunomodulatory effects by suppressing $\mathrm{T}$ cells and other lymphocytes. Emerging reports on cell-based clinical studies in regenerative medicine indicate that mesenchymal stem cells are the most commonly applied cells in the phase three of clinical trials. However, it should be noted that fibroblasts, as end-stage differentiated cells, are much safer therapeutic products for the market.

\section{References}

1. Shi C, Zhu Y, Su Y, Cheng T. Stem cells and their applications in skin-cell therapy. Trends Biotechnol. 2006;24(1):48-52. doi: 10.1016/j.tibtech.2005.11.003. [PubMed:16298447].

2. Sung HM, Suh IS, Lee HB, Tak KS, Moon KM, Jung MS. Case Reports of Adipose-derived Stem Cell Therapy for Nasal Skin Necrosis after Filler Injection. Arch Plast Surg. 2012;39(1):51-4. doi: 10.5999/aps.2012.39.1.51. [PubMed: 22783492]. [PubMed Central: PMC3385290].

3. Mansbridge J. Skin tissue engineering. J Biomater Sci Polym Ed. 2008;19(8):955-68. doi: 10.1163/156856208784909417. [PubMed: 18644224].

4. Rawlings AV. Ethnic skin types: are there differences in skin structure and function? Int J Cosmet Sci.2006;28(2):79-93. doi:10.1111/j.14672494.2006.00302.x. [PubMed: 18492142].

5. Montagna W. The structure and function of skin. Elsevier; 2012.

6. Honari G. Skin structure and function. Sensitive Skin Syndrome. Second ed. CRC Press; 2017. p. 26-32.

7. McGrath JA, Eady RA, Pope FM. Anatomy and organization of human skin. Rook's textbook of dermatology. 3. 2004. p. 1-5.

8. Metcalfe AD, Ferguson MW. Tissue engineering of replacement skin: the crossroads of biomaterials, wound healing, embryonic development, stem cells and regeneration. J R Soc Interface. 2007;4(14):41337. doi: 10.1098/rsif.2006.0179. [PubMed: 17251138]. [PubMed Central: PMC2373411].

9. Polakowska RR, Piacentini M, Bartlett R, Goldsmith LA, Haake AR. Apoptosis in human skin development: morphogenesis, periderm, and stem cells. Dev Dyn. 1994;199(3):176-88. doi: 10.1002/aja.1001990303. [PubMed: 7517223].

10. Driskell RR, Lichtenberger BM, Hoste E, Kretzschmar K, Simons $\mathrm{BD}$, Charalambous $\mathrm{M}$, et al. Distinct fibroblast lineages determine dermal architecture in skin development and repair. Nature. 2013;504(7479):277-81. doi: 10.1038/nature12783. [PubMed: 24336287]. [PubMed Central: PMC3868929].

11. Harper RA, Grove G. Human skin fibroblasts derived from papillary and reticular dermis: differences in growth potential in vitro. Science. 1979;204(4392):526-7. doi: 10.1126/science.432659. [PubMed: 432659].
12. Schafer IA, Pandy M, Ferguson R, Davis BR. Comparative observation of fibroblasts derived from the papillary and reticular dermis of infants and adults: Growth kinetics, packing density at confluence and surface morphology. Mech Ageing Dev.1985;31(3):275-93. doi: 10.1016/0047-6374(85)90095-8.

13. Sorrell JM, Baber MA, Caplan AI. Site-matched papillary and reticular human dermal fibroblasts differ in their release of specific growth factors/cytokines and in their interaction with keratinocytes. J Cell Physiol. 2004;200(1):134-45. doi: 10.1002/jcp.10474. [PubMed: 15137066].

14. Phan SH. Biology of fibroblasts and myofibroblasts. Proc Am Thorac Soc. 2008;5(3):334-7. doi: 10.1513/pats.200708-146DR. [PubMed: 18403329]. [PubMed Central: PMC2645244].

15. Bayreuther K, Rodemann HP, Hommel R, Dittmann K, Albiez M, Francz PI. Human skin fibroblasts in vitro differentiate along a terminal cell lineage. Proc Natl Acad Sci U S A. 1988;85(14):5112-6. doi: 10.1073/pnas.85.14.5112. [PubMed: 3393534]. [PubMed Central: PMC281698].

16. Blau HM, Pavlath GK, Hardeman EC, Chiu CP, Silberstein L, Webster SG, et al. Plasticity of the differentiated state. Science. 1985;230(4727):75866. doi: 10.1126/science.2414846. [PubMed: 2414846].

17. Howling GI, Dettmar PW, Goddard PA, Hampson FC, Dornish M, Wood EJ. The effect of chitin and chitosan on the proliferation of human skin fibroblasts and keratinocytes in vitro. Biomaterials. 2001;22(22):2959-66. doi: 10.1016/S0142-9612(01)00042-4. [PubMed: 11575470].

18. Varani J, Dame MK, Rittie L, Fligiel SE, Kang S, Fisher GJ, et al. Decreased collagen production in chronologically aged skin: roles of age-dependent alteration in fibroblast function and defective mechanical stimulation. Am J Pathol. 2006;168(6):1861-8. doi: 10.2353/ajpath.2006.051302. [PubMed: 16723701]. [PubMed Central: PMC1606623].

19. Sorrell JM, Caplan AI. Fibroblast heterogeneity: more than skin deep. J Cell Sci. 2004;117(Pt 5):667-75. doi: 10.1242/jcs.01005. [PubMed: 14754903].

20. Halfon S, Abramov N, Grinblat B, Ginis I. Markers distinguishing mesenchymal stem cells from fibroblasts are downregulated with passaging. Stem Cells Dev. 2011;20(1):53-66. doi: 10.1089/scd.2010.0040. [PubMed: 20528146].

21. Wagner W, Wein F, Seckinger A, Frankhauser M, Wirkner U, Krause U, et al. Comparative characteristics of mesenchymal stem cells from human bone marrow, adipose tissue, and umbilical cord blood. Exp Hematol. 2005;33(11):1402-16. doi: 10.1016/j.exphem.2005.07.003. [PubMed: 16263424]. 